\title{
Empirical Evidence during the Implementation of an Educational Chatbot with the Electroencephalogram Metric*
}

\author{
Andreia Solange Bos ${ }^{1}$ (), Michelle Câmara Pizzato' (), Marcelo Vettori ${ }^{2}$ (),

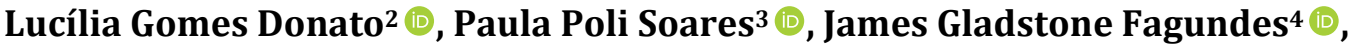 \\ Milton Antônio Zaro 5 (i)



How to cite this paper: Bos, A. S., Pizzato, M. C., Vettori, M., Donato, L. G., Soares, P. P., Fagundes, J. G., \& Zaro, M. A. (2020). Empirical Evidence during the Implementation of an Educational Chatbot with the Electroencephalogram Metric. Creative Education, 11, 2337-2345.

https://doi.org/10.4236/ce.2020.1111171

Received: September 10, 2020

Accepted: November 21, 2020

Published: November 24, 2020

Copyright $\odot 2020$ by author(s) and Scientific Research Publishing Inc. This work is licensed under the Creative Commons Attribution International License (CC BY 4.0).

http://creativecommons.org/licenses/by/4.0/

\begin{abstract}
A student's attention status during a digital conversation can differ when talking to an intelligent agent, that is, a chatterbot interactive. There is evidence that brain waves have different oscillations during the study. A virtual platform with an artificial intelligence chatterbot was explored in this study. During the study, participants were asked to use the Neurosky Mindwave sensor to assess their attention levels in real time. Statistical analyses of the results revealed significant effects on the participants' attention levels. The chatbot was associated with dominant proportions of delta and theta brain waves in didactic content. Conversation via social interaction chatbot triggered dominant gamma brain waves.
\end{abstract}

\section{Keywords}

Chatbot, Attention, Eeg, Neurosky

\section{Introduction}

The virtual world is becoming part of our everyday life. It is essential that there is the development of tools and applications that allow us to live in this human condition, adapting to changes in the areas of technology. The effective interac-

${ }^{\star}$ Empirical evidence chatbot. 
tion between computers and humans is expected to be increasing and is increasing exponentially (Bos et al., 2019a). Established technologies like personal computer and internet access have become almost ubiquitous for learning. In addition, relatively new technologies appear every moment in the globalized world, an example is the study that we will be presenting in this article, in which we will use the chatbot technology and the measurement sensor of the participant's brain activity sample.

AI-mediated communication represents a new paradigm where communication is generated by an intelligent system. Spell checking, texts are used for clarity in communication. A chatbot follows the flow shaped by the programmer in order to produce an effective dialogue using questions and answers in dialog format. It is important to determine what kind of questions to ask, what information should be provided to process responses to the user, a flow is based on a cycle formed by the bot's message (questions); the user's message (reply); and decision making (response processing). A conversation strategy should focus on recommendation tasks. Teaching is an act based on communication and interaction, and chatbots have significant educational potential in their communication skills through the language of a system with a knowledge base.

It is increasingly necessary to meet the needs of apprentices. Teachers need to start from the premise that education must constantly dialogue with neuroscience, thus, generating an understanding that evidences brain activity during the process as a whole. Neuroeducation is the area of research that aims to link educational technologies with students' cognitive skills and their specificities (Tokuhama-Espinosa, 2008).

The lack of opportunities for engagement, professional training and incentives for teachers in Brazil is the consequences in which teachers still lack the appropriate technologies (Bos et al., 2019b), always considering that the success and effectiveness of the implementation of educational technology are largely linked to the positive perceptions of teachers. Measures could be taken to increase teachers' willingness to adopt such technologies in their teaching practices.

In this context, this work presents a study that brings new trends in tools and platforms, using the Zenvia platform as a reference used during the study.

\section{Theoretical Framework}

As a result of conducting an educational survey, we have found that NeuroSky has been widely applied to several research topics. It can be used in teaching and learning; the following will mention some related works already developed with chatterbots in education and the use of the sensor to collect evidence in brain activities.

With the study of learning materials, it was possible to find the works of Shadiev et al., 2017b, who adopted the EEG to investigate the effectiveness of text speech recognition applications in learning performance, attention and student meditation. 
Google Home, is a smart speaker made for Google, which answers voice commands, its software is based on the Google Assistant chatbot. It allows the user several commands such as: checking the time, a search with music, videos, etc. Some frameworks have been proposed to facilitate the development of chatbots (Yan et al., 2016).

IBM Watson Assistant is an example of a bot with many abilities. The user can open an account and experience a chat with the virtual assistant that he will quickly have many answers to his questions.

A chatbot called ELIZA was created in 1966 at MIT to imitate human conversation and simulate a physical therapist in clinical treatment (Abu Shawar et al., 2007).

The ALICE chatbot was created in 1995 and was inspired by ELIZA. ALICE was conceived as a conversational agent and at the time many people thought they were talking to a real human being during interactions.

Chatbots are used daily by some people. Apple's Siri, Amazon's Alexa, Microsoft Cortana and Samsung's Bixby have the ability to open applications, play music and be a virtual assistant (Dale, 2016).

Bos et al., conducted a study on the use of the Neurosky sensor to measure students' attention by measuring interactivity with the interactive video platform and identifying the stimulus response (Bos et al., 2019c; Bos et al., 2020; Bos et al., 2019e: p. 3).

Shi mentions that correctly inferring user preferences for items is crucial for effective recommendations (Shi, Larson, \& Hanjalic, 2014).

Recently, rapid progress in technology has transformed computer-mediated communication by artificial intelligence-mediated communication, which is robust and has been widely used (Hohenstein \& Jung, 2020). As technologies evolve, they become readily available and are adapted to the areas of education. Technological innovations can increase student engagement and motivation. Whereas a chatbot offers a range of potential benefits, including personalization and instant availability 24 hours a day, 7 days a week. These positive aspects are interesting in the education sector. This branch of research is only emerging in the scientific community, so in our article we propose this new technology for educational use in addition to the business that has been in use for a longer time.

In contrast to previous studies, we focus on a mechanism that alternates between questions and recommendations, in addition we use the method of adjusting the parameters they have in interactions. Thus, introducing a bot and user conversation model, simulating the systematic interactions inferred in a knowledge base with didactic content and social interactions.

\section{Methodology}

In order to establish an empirical basis from which design principles for educational chatbots derive, we explored the previous studies. So, we started implementing a bot suitable for the educational context.

This work shows an experience carried out with student volunteers, exploring 
the impacts of using a platform for creating and implementing chatbots during the teaching process, with a total of 7 participants. In this scenario, the production of digital content previously prepared was exposed to participants on the platform. In the implementation, we used the Zenvia tool, which is a development platform for creating chatbots with human computer interaction technology based on natural language processing. The questions were divided into categories of activities, social interactions, realist, didactic and conventional content.

\subsection{Materials and Methods}

In this study, the participants talked with the chatbot in AI format simulating a dialogue with a virtual teacher with questions and answers. During the virtual conversation, the participant was controlled by a sensor to capture samples of brain activities. According to the user's responses, the ultimate goal of the chatbot is to allow him to respond appropriately to the student's questions and interactions (Bos et al., 2019c). There were two moments of studies carried out in which the interactions with the chatbot occurred. At first, the participants interacted with the chatbot with social interactions in which there were interactions such as "Hello, what's your name"; "how are you today"; "where do you live"; "What you like to do" among other social interactions. In the second moment of the study, the participants were invited to interact with the chatbot with didactic content about general knowledge.

\subsection{The Zenvia Conversation Platform}

The dialogue platform mediated by AI (Artificial Intelligence) used in this study was the Zenvia Conversational Cloud. After selecting the creation of a new chatbot, the "bot design" screen used to build the conversation flow was used. The stream screen has start and end chatbot balloons on a connection line. The inclusion of the interaction steps occurs between "sends message" webchat channels and "receives message" and after decision point. When a user answers a multiple-choice question, using an interface is more convenient than free text input. Still, the system needs to provide answer options in addition to the questions. Figure 1 shows the flowchart of the proposed dialogue for decision-making points.

\subsection{The Neurosky Mindwave Sensor}

The NeuroSky MindWave Mobile sensor (NeuroSky Inc., San Jose, CA, USA, MindWave) is one of the most popular and affordable EEG devices. In addition, the current trend of adopting this device makes it essential to help researchers and end users to understand its validity (Bos et al., 2019d). MindWave developers claim to be able to measure cognitive functions, such as states of attention and relaxation, with just a passive dry electrode on the forehead, located in the Fp1 (left frontal lobe). To collect brain frequencies, the Neuroexperimenter

software installed on the computer was used to collect data. Figure 2 shows the Neuroexperimenter and Neurosky software. 


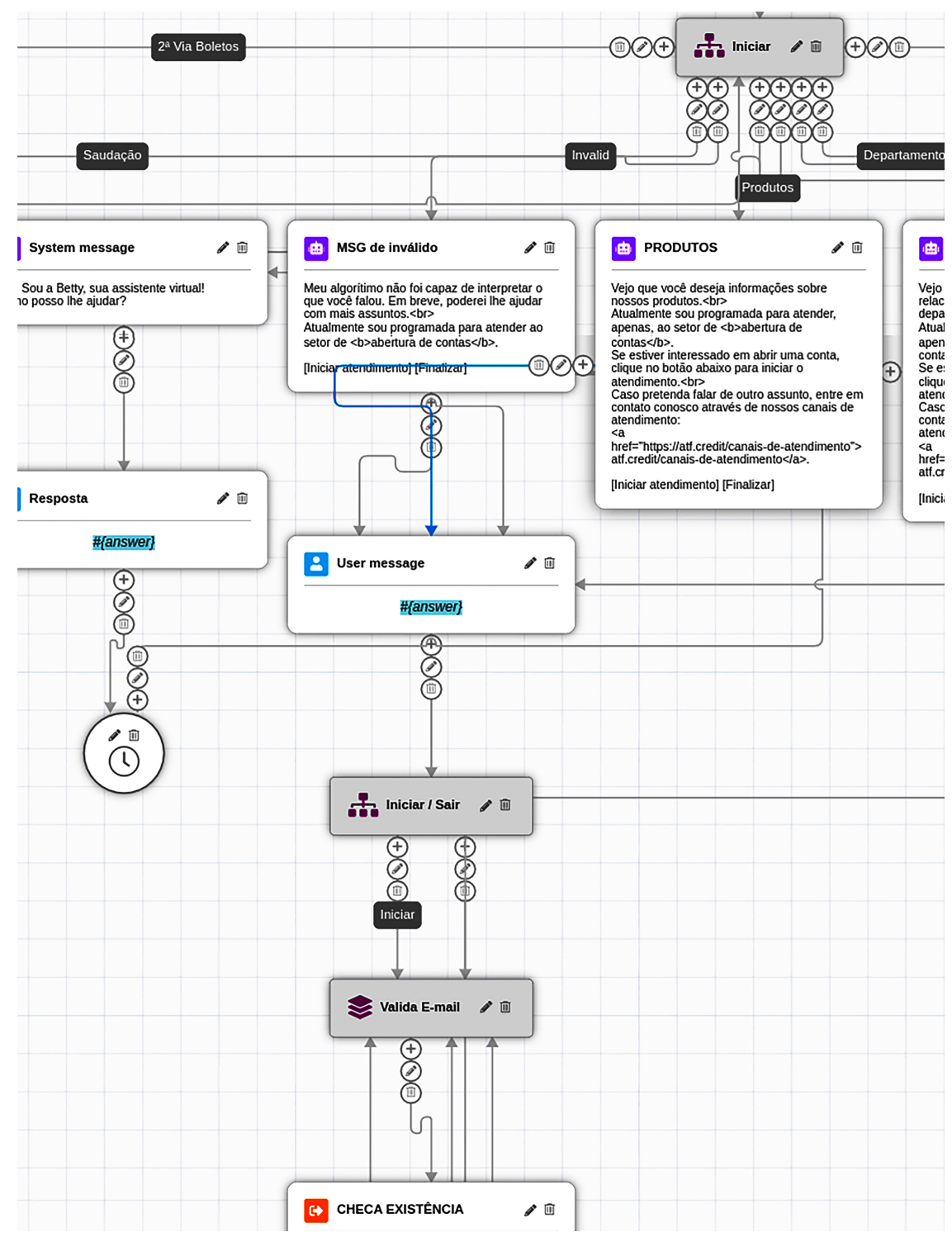

Figure 1. Flowchart of the dialogue, points of decision making.

\section{Results}

To solve the problem of better student productivity, it was necessary to create and develop the chatbot where the developer focused on creating the logical structure. Technology like Zenvia's platform helps to reduce teachers' work, replacing student questions and feedback with artificial intelligence. Chatbot is helping to reduce faculty work by using chatbot and text analysis technology to assess student assignments and tests. In study 1 (Figure 3 and Figure 4), the analysis of responses is presented, in which the participants were monitored with their brain activities during the conversation with the chatbot with social interactions. Figure 3 and Figure 4 show the analysis of responses during the conversation with the social interaction chatbot.

It is noticed that in the trend line for attention, concentration occurs with the highest potential coefficient of determination for the attention spectrum with a 


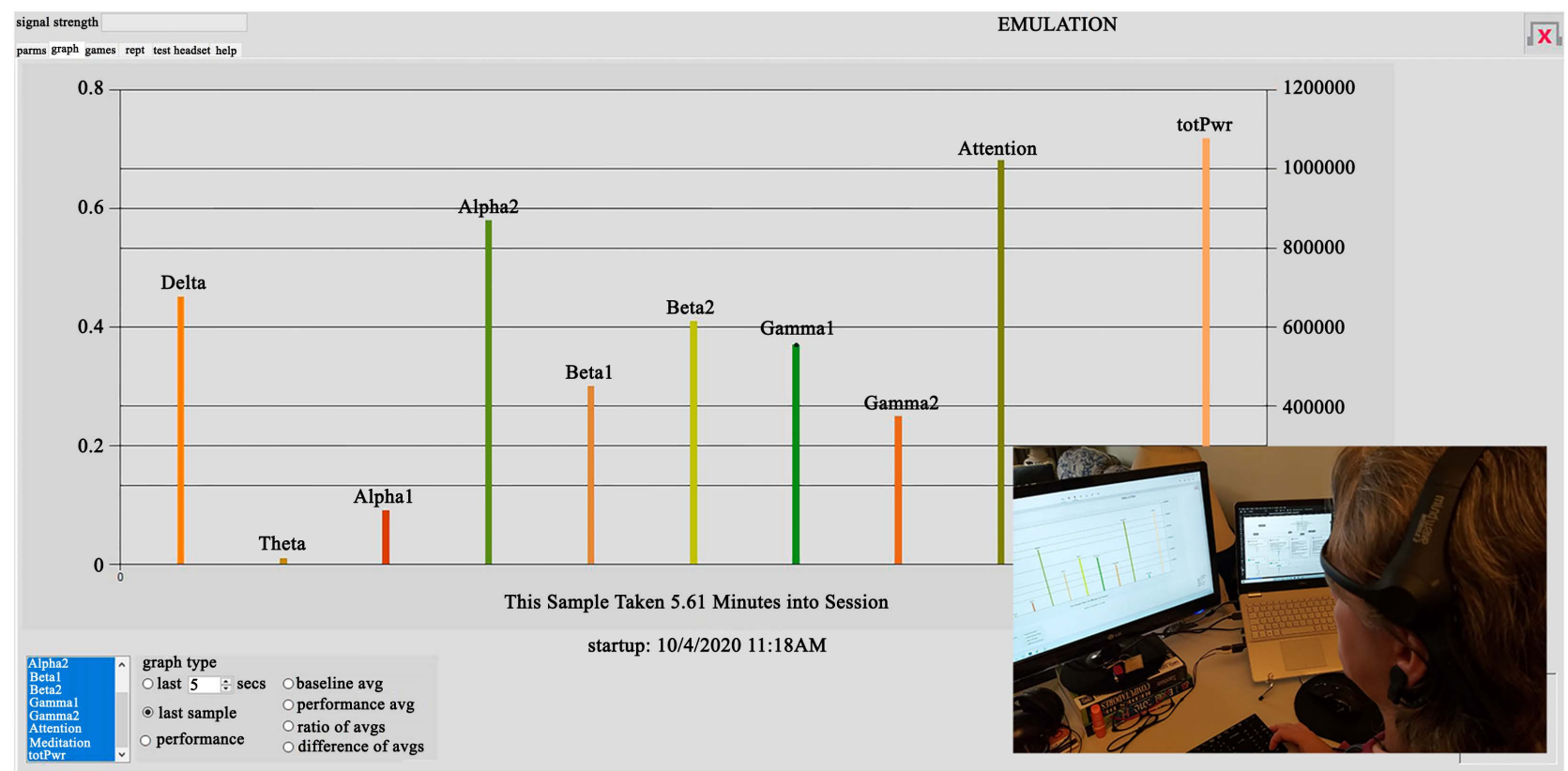

Figure 2. Neuroexperimenter in use with the Neurosky sensor. Source: by the author, 2020.

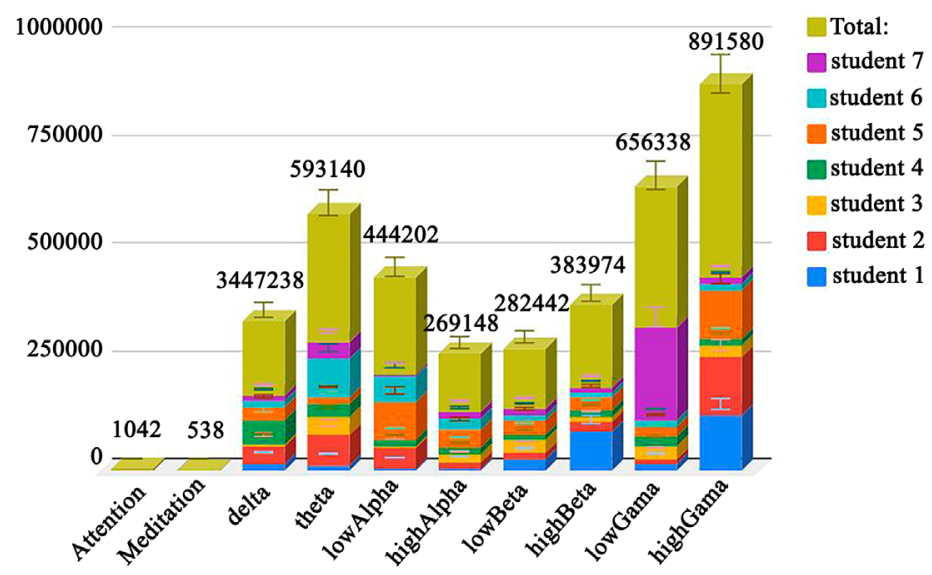

Figure 3. Analysis of responses during the conversation with the social interaction chatbot.

\section{Attention e Meditation}

Attention - Linha de tendência para Attention $\mathrm{R}^{2}=0.011$

Meditation $=$ Linha de tendência para Meditation $\mathbf{R}^{2}=0.06$

125

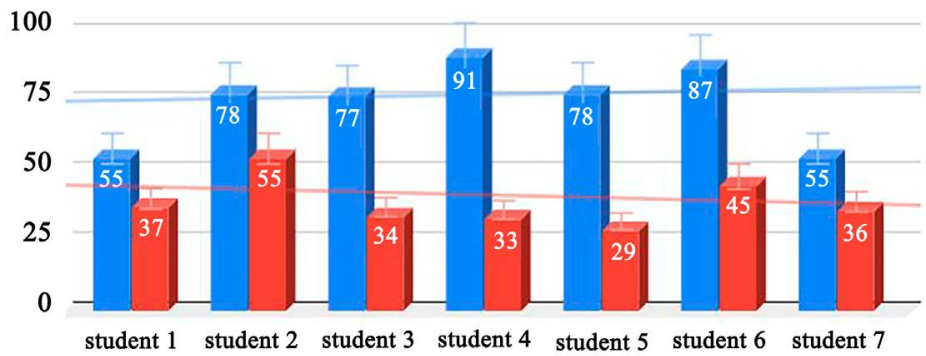

Figure 4. Analysis of attention during the conversation with the chatbot. 
$\mathrm{R}^{2}$ score of 0.011 in the color shown in blue. In red, it is possible to observe the trend line for meditation at $\mathrm{R}^{2} 0.06$. Also, in this data set, the brain frequencies that most oscillated positively during the study were measured, which were the low Gama and high Gama waves. The low Gama and high Gama waves work at a frequency of (30 to $50 \mathrm{~Hz}$ ) with multisensory processing and act on complex cognitive functions. Still for the attentional spectrum in Figure 3, a score greater than 1042 is perceived for attention in relation to meditation with a score of 530 points.

In study 2 (Figure 5 and Figure 6), the analysis of responses is presented, in which the participants were monitored with their brain activities during the conversation with the chatbot with the interactions of general knowledge with didactic content. Figure 5 and Figure 6 show the analysis of responses during the conversation with the chatbot.

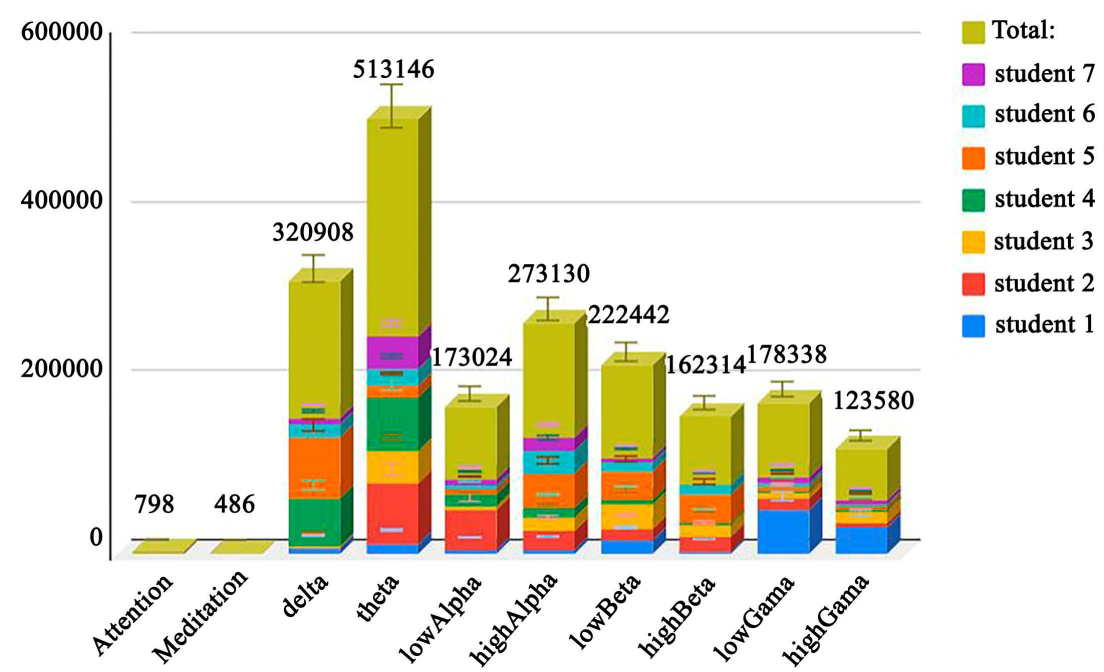

Figure 5. Analysis of responses during the conversation with the chatbot of didactic content.

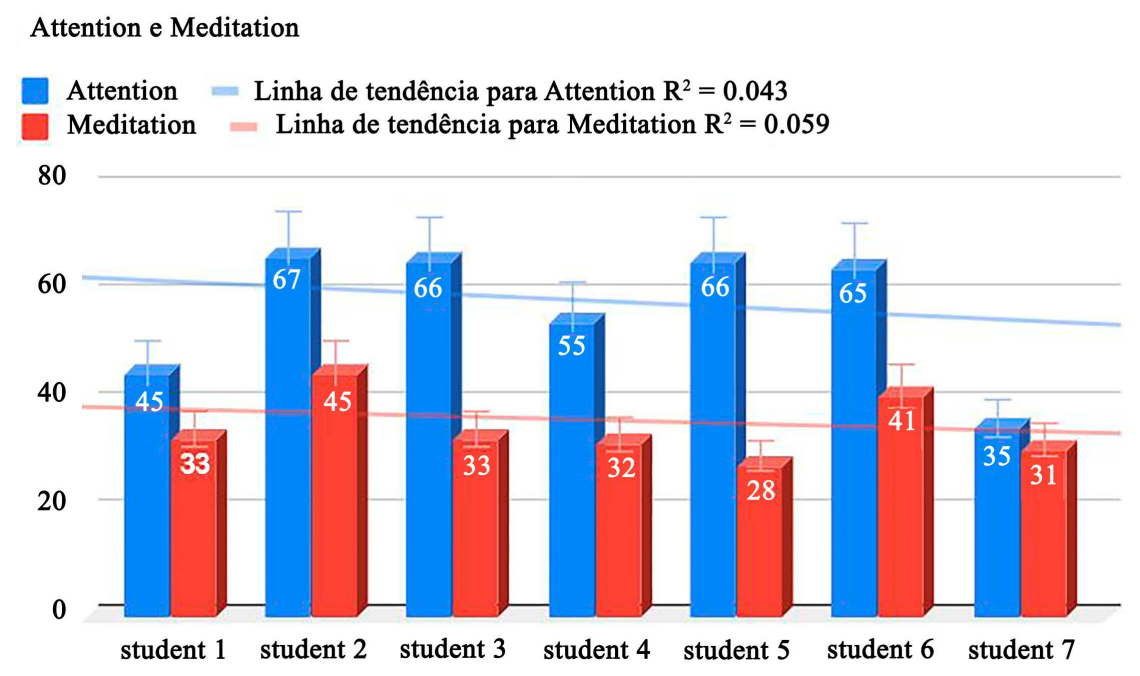

Figure 6. Analysis of student attention in decision making with educational content. 
The most dominant brain waves in this study were the delta and theta waves, which are electromagnetic frequencies provided by brain electrical activity. The Delta wave ( 1 to $3 \mathrm{~Hz}$ ) refers to the states of mind of expanded consciousness. The Theta wave is the state of meditation, intuition, creativity and memory. The Theta wave is in a frequency range ( 4 to $8 \mathrm{~Hz}$ ). It is possible to show that the sample shown in Figure 5 has its frequency range of attention at 798 points. Already followed by meditation at 486 points. Still during the decision-making with the educational content, the trend line among the participants had $\mathrm{R}^{2}$ of 0.043 and $\mathrm{R}^{2} 0.0059$ for meditation, thus showing the differences in measures between the comparative studies.

\section{Conclusion}

This research brought the research approach on systemic neuroscience, in which, the objective was to expose some experiences of neuroscience in the teaching process, studying the regions of the brain in the processes of measures evoked from attention spectra.

The diagram of the operational flow of the chatbot, to the questions proposed by the bot in Portuguese language, refers to the educational content approached according to the theme proposed by the teacher, together with the social interactions compared here in the study. The results obtained in the study are satisfactory and show a good perspective on this type of educational approach.

Teachers still find it difficult to produce interactive educational resources, as in some situations it is necessary to have prior knowledge in programming languages and web development. Therefore, the platform like Zenvia can be a possibility, as it does not require advanced knowledge and is an attractive educational resource for teachers. It is still an emerging technology that with distance learning teaching will be increasingly in evidence and with more advanced artificial intelligence techniques.

This study has some limitations, although some articles have educational contexts, they are mostly not for educational purposes. However, this study sought to investigate the area of educational chatbot. To develop a chatbot with various purposes and functions for educational purposes, it is necessary to make several efforts with more specialists.

Subsequent developments involve the application of other approaches proposed by more teachers in various contexts and an improvement in the online platform to make it more robust in its knowledge base.

\section{Acknowledgements}

Federal Institute of Rio Grande do Sul (IFRS), Brazil.

Federal University of Rio Grande do Sul (UFRGS), Brazil.

Pontifical Catholic University of Rio Grande do Sul (PUC-RS), brazil.

John XXIII College of Rio Grande do Sul, Porto Alegre-RS, Brazil.

National Commercial Learning Service, (SENAC-RS), Porto Alegre-RS, Brazil. 


\section{Conflicts of Interest}

The authors declare no conflicts of interest regarding the publication of this paper.

\section{References}

Abu Shawar, B., \& Atwell, E. (2007). Chatbots: Are They Really Useful? LDV-Forum Band, 22, 29-49.

Bos, A. S. et al. (2019b). Student's Attention: The Use of Brain Waves Sensors in Interactive Videos. International Journal of Advanced Engineering Research and Science, 6, 155-157. https://doi.org/10.22161/ijaers.6.4.18

Bos, A. S., Donato, L. G., Vettori, M., \& Zaro, M. A. (2020). Effects of the Binaural Wave as a Stimulus for Student Hyperattention: Brain Frequency Records without Interactive Media Context. International Journal of Advanced Engineering Research and Science, 7, 208-213. http://journal-repository.com/index.php/ijaers/article/view/2491 https://doi.org/10.22161/ijaers.79.24

Bos, A. S., Herpich, F., Kuhn, I., Guarese, R. L. M., Tarouco, L. M. R., Zaro, M. A., \& Wives, L. (2019a). Educational Technology and Its Contributions in Students' Focus and Attention Regarding Augmented Reality Environments and the Use of Sensors. Journal of Educational Computing Research, 57, 1832-1848. https://doi.org/10.1177/0735633119854033

Bos, A. S., Pizzato, M. C., \& Zaro, M. (2019c). An Experiment to Measure the Level of Student Attention: The Use of Interactive Media as a Stimulus Response. RENOTE-Magazine New Technologies in Education, 17, No. 3.

Bos, A. S., Pizzato, M., \& Zaro, M. A. (2019e). Investigation of Student Attention: The Use of Virtual Reality in Computing Education. Tear: Journal of Education, Science and Technology, 8, 3. https://periodicos.ifrs.edu.br/index.php/tear/index https://doi.org/10.35819/tear.v8.n2.a3586

Bos, A. S., Zaro, M. A., \& Pizzato, M. (2019d). Investigation of Student Attention with EEG techniques: The Use of Virtual Reality in Teaching. In Proceedings of the Workshops Brazilian Congress of Informatics in Education (p. 1397).

Dale, R. (2016). The Return of Chatbots. Natural Language Engineering, 22, 811-817. https://doi.org/10.1017/S1351324916000243

Hohenstein, J., \& Jung, M. (2020). AI as a Moral Crumple Zone: The Effects of AI-Mediated Communication on Attribution and Trust. Computers in Human Behavior, 106, 106190. https://doi.org/10.1016/j.chb.2019.106190

Shadiev, R., Wu, T.-T., \& Huang, Y.-M. (2017b). Enhancing Learning Performance, Attention, and Meditation Using a Speech-to-Text Recognition Application: Evidence from Multiple Data Sources. Interactive Learning Environments, 25, 249-261.

https://doi.org/10.1080/10494820.2016.1276079

Shi, Y., Larson, M., \& Hanjalic, A. (2014). Collaborative Filtering beyond the User Item Matrix: Research on the State of the Art and Future Challenges. ACM Computing Surveys, 47, Article No.: 3. https://doi.org/10.1145/2556270

Tokuhama-Espinosa, T. N. (2008). The Scientifically Substantiated Art of Teaching: A Study in the Development of Standards in the New Academic Field of Neuroeducation (Mind, Brain, and Education Science). Capella University, MN, USA.

Yan, M., Castro, P., Cheng, P., \& Ishakian, V. (2016). Building a Chatbot with Serverless Computing. In Proceedings of the 1st International Workshop on Mashups of Things and APIS (pp. 5:1-5:4). New York: ACM. https://doi.org/10.1145/3007203.3007217 